\title{
A pilot practice-based outcomes evaluation of low- intensity cognitive behavioural interventions delivered by postgraduate trainees to children and young people with mild to moderate anxiety or low mood: an efficient way forward in mental health care?
}

\author{
Gavin Lockhart ${ }^{1 \star}$, Christina Jones ${ }^{2}$ and Victoria Sopp ${ }^{1}$ \\ ${ }^{1}$ Mental Health Practice Unit, University of Sussex, UK and ${ }^{2}$ School of Psychology, University of Surrey, UK \\ ${ }^{*}$ Corresponding author. Email: Gavin.Lockhart1@nhs.net
}

(Received 1 October 2020; revised 10 September 2021; accepted 13 September 2021)

\begin{abstract}
Anxiety and depression affect a significant number of children and young people (CYP) and can have a far reaching and long-lasting impact. Cognitive behavioural $(\mathrm{CB})$ interventions can be effective for treating anxiety and depression in CYP but are difficult to access. Recent government policy in England seeks to train a non-traditional graduate workforce to deliver a range of $\mathrm{CB}$ interventions for mild to moderate anxiety and depression to CYP, in community settings. This practice-based evaluation aimed to estimate the effectiveness of $\mathrm{CB}$ interventions delivered by postgraduate trainees undertaking training in a range of $\mathrm{CB}$ interventions for mild to moderate anxiety and depression whilst on placement in schools or community Child and Adolescent Mental Health Services (CAMHS). Self- and parentreported routine outcome measures (ROMS) were completed pre- and post-intervention, including measures of symptom severity, symptom impact and goal achievement. Significant improvements were demonstrated across all self and parent-reported measures post-intervention, with mean scores falling firmly in the non-clinical range, a significant reduction in the proportion of CYP in the clinical range on measures, and predominantly medium to large effect sizes. Results are promising in terms of the capacity to train a graduate workforce to deliver a range of low-intensity CB interventions to CYP experiencing mild to moderate depression or anxiety-based difficulties in either CAMHS or school settings, increasing capacity across the system. The current practice-based evaluation also supports the potential effectiveness of current training models/programmes. Further research is needed in terms of long-term outcomes and to compare outcomes between settings, interventions, and demographic groups.
\end{abstract}

\section{Key learning aims}

(1) To understand the potential prevalence rates of mental health difficulties in children and young people in England, and their wider impact.

(2) Critical awareness of the evidence base for cognitive and behavioural interventions for depression and anxiety in children and young people.

(C) The Author(s), 2021. Published by Cambridge University Press on behalf of the British Association for Behavioural and Cognitive Psychotherapies. This is an Open Access article, distributed under the terms of the Creative Commons Attribution licence (https:// creativecommons.org/licenses/by/4.0/), which permits unrestricted re-use, distribution, and reproduction in any medium, provided the original work is properly cited. 
(3) Awareness of gaps in access to evidence-based psychological interventions for children and young people in England.

(4) Knowledge of emerging graduate-level low-intensity psychological practitioner roles in England.

(5) Awareness of the emerging evidence base for the estimated effectiveness of low-intensity cognitive and behavioural interventions delivered to children and young people by graduate-level practitioners in schools and community mental health settings.

Keywords: anxiety; behavioural; children; cognitive; depression; low-intensity

\section{Introduction}

\section{Prevalence of mental health difficulties in children and young people}

Rates of mental health difficulties in children and young people (CYP) are high. Estimates suggest that between 10 and 50\% of CYP meet diagnostic criteria for a mental health disorder at any time (Collins et al., 2011; Green et al., 2004; Harden et al., 2001). NHS Digital data in 2018 (Sadler et al., 2018 ) estimated that one in eight 5- to 19-year-olds (12.5\%) in England experienced at least one mental health disorder, although the most recent NHS Digital data (Vizard et al., 2020) suggested that this had risen to one in six CYP (16.7\%).

Anxiety and depression are amongst the most common mental health difficulties experienced by CYP (Green et al., 2004; Sadler et al., 2018; Vizard et al., 2020). Between 4 and 7\% of CYP meet criteria for an anxiety disorder, increasing to a cumulative frequency of $10 \%$ by 16 years of age (Ford et al., 2003). Depression affects around 3-6\% of CYP, also increasing with age (Costello et al., 2006).

Whilst the impact of the COVID-19 pandemic and associated safety measures on the mental health of CYP is not fully known, and studies are ongoing, there are early indications and widespread consensus that it is likely to lead to a further rise in mental health difficulties for CYP (Banks and Xu, 2020; Bignardi et al., 2020; Fancourt et al., 2021; Levita et al., 2020; Raw et al., 2021; Shum et al., 2021; Vizard et al., 2020; Waite et al., 2020).

\section{Psychological interventions for anxiety and depression in CYP}

Findings from multiple systematic reviews conclude that there is strong evidence for the effectiveness of cognitive behavioural (CB) interventions in treating anxiety in CYP (Cartwright-Hatton et al., 2004; Ewing et al., 2015; Fonagy et al., 2015; James et al., 2020; Wang et al., 2017; Warwick et al., 2017). CB interventions are defined as a psychological model of treatment involving core components of identifying and modifying unhelpful cognitions and/or modifying unhelpful behavioural responses (e.g. Caldwell et al., 2019; James et al., 2020). A recent Cochrane review found that, following CB interventions, approximately $50 \%$ of CYP recover from their primary anxiety disorder (James et al., 2020), and between 47 and $66 \%$ are in remission from all anxiety disorders, compared with 19-21\% of waitlist controls (James et al., 2020; Warwick et al., 2017). There is also evidence for the sustainability of improvements at 6- and 12-month follow-up (Fonagy et al., 2015; James et al., 2020). Studies have suggested that interventions including a cognitive component are effective for generalised anxiety, separation anxiety and social phobia (Fonagy et al., 2015), whereas behavioural-based exposure treatment is more effective for specific phobias and school refusal (Roth and Fonagy, 2005). To date there is only tentative evidence for the effectiveness of CB interventions delivered to CYP with milder or non-clinical anxiety symptoms, with metaanalyses finding small to no effects (Caldwell et al., 2019; Johnstone et al., 2018; Rasing et al., 2017; Stockings et al., 2016; Werner-Seidler et al., 2017). This includes manualised programmes such as the classroom-based FRIENDS programme (Liddle and Macmillan, 2010; Stallard et al., 2014) and Cool Kids (McLoone and Rapee, 2012; Mifsud and Rapee, 2005). A recent network meta-analysis on preventative and targeted school-based interventions 
concluded that there was weak evidence for CB interventions reducing anxiety symptoms across both primary and secondary age CYP (Caldwell et al., 2019). Caldwell et al. (2019) also found insufficient evidence for other school-based wellbeing approaches such as interpersonal therapy (IPT), occupational therapy, and attention control. CB interventions can therefore be highly effective in the treatment of anxiety in CYP but require further study in relation to milder or sub-clinical anxiety and when delivered in community settings.

With respect to depression, meta-analyses and large-scale treatment trials have also shown $\mathrm{CB}$ interventions to be an effective psychological approach for treating CYP with depression, with 40$60 \%$ response rates and evidence of sustained improvements (Eckshtain et al., 2020; March and Vitiello, 2009; Munoz-Solomondo et al., 2008; Oud et al., 2019; Weisz et al., 2006). Recent studies and meta-analyses have shown promising findings with respect to behavioural activation adapted for CYP (Brett et al., 2020; Kitchen et al., 2020; Martin and Oliver, 2019; McCauley et al., 2011; McCauley et al., 2016; Pass et al., 2015; Pass et al., 2016; Pass et al., 2018b; Tindall et al., 2017). Positive outcomes have been demonstrated for behavioural activation delivered to CYP in the UK across both clinical (Kitchen et al., 2020; Pass et al., 2015; Pass et al., 2016; Pass et al., 2018b) and school settings (Arnott et al., 2020; Brett et al., 2020; Pass et al., 2018c). Regarding treatment efficacy, studies have suggested that interventions with a cognitive component are no more effective than behavioural interventions for depressive symptoms in CYP (Weisz et al., 2006), although there is recent evidence to suggest that treatment combining elements of behavioural activation with challenging thoughts (as part of cognitive restructuring) is associated with improved outcomes (Oud et al., 2019). As with studies of anxiety, meta-analyses of CB interventions delivered to CYP with milder depression or sub-clinical symptoms of depression show mixed results (Asarnow et al., 2001; Johnstone et al., 2018; Rasing et al., 2017; Stockings et al., 2016; Werner-Seidler et al., 2017). A recent network meta-analysis on preventative and targeted school-based interventions concluded that there was no evidence for CB interventions reducing depressive symptoms in CYP (Caldwell et al., 2019), although this contradicts the encouraging findings of a previous meta-analysis (Calear and Christensen, 2010) and the more recent studies of behavioural activation. $\mathrm{CB}$ interventions can therefore be effective in the treatment of depression in CYP but show mixed results in relation to milder or sub-clinical depression and when delivered in community settings, which requires further study. Recent studies noted above suggest that behavioural activation may be a more effective CB intervention compared with other $\mathrm{CB}$ interventions (e.g. cognitive restructuring) in these circumstances.

\section{The need for quick and efficient support}

Mental health difficulties in childhood can have a significant short- and long-term impact on a young person's life, including reduced educational attainment and job prospects, and an increased risk of criminality, self-harm, addictions, and sexually vulnerable behaviour (Department of Health CYP Mental Health \& Wellbeing Taskforce, 2015; Essau et al., 2014; Murphy and Fonagy, 2012). In addition, studies have identified that $50 \%$ of mental health problems in adulthood begin in childhood, with over $75 \%$ starting before the age of 18 (Kessler et al., 2005; Kessler et al., 2007; Kim-Cohen et al., 2003). The case for early and effective intervention for CYP experiencing mental health difficulties is therefore compelling.

\section{Access to evidence-based support}

Despite the argument for early intervention, and the existence of effective psychological interventions for anxiety and depression in CYP, it has been estimated that less than $25-35 \%$ of CYP with diagnosable mental health conditions access support (Andrews et al., 2002; Care Quality Commission, 2018; Green et al., 2004; House of Commons Health Committee, 2014; National Institute for Health \& Care Excellence, 2019; NHS, 2019; Rees et al., 2008). 
Although anxiety is the most prevalent mental health difficulty in CYP, and one of the most treatable, it has historically been the least treated (Ford et al., 2007). Parents have reported perceived barriers to seeking and accessing support, such as not knowing who to ask for help or what help is available, as well as subsequent difficulties with getting referrals, and long waiting times (Reardon et al., 2019). Few of those CYP who do receive support for mental health difficulties have access to a full range of evidence-based interventions (Fonagy et al., 2015; Independent Mental Health Taskforce, 2016). A recent UK study found that although $65 \%$ of parents of CYP with an anxiety disorder reported contacting a professional for help or advice, less than $40 \%$ received any professional help, and less than 3\% received evidence-based $\mathrm{CB}$ interventions, with the majority receiving counselling, parenting support, or signposting to other self-help resources (Reardon et al., 2019).

Average waiting times for access to psychological therapy in CYP mental health services in the UK are also long (Department of Health CYP Mental Health \& Wellbeing Taskforce, 2015; Frith, 2017; NHS Digital, 2020). For example, a Department of Health CYP Mental Health \& Wellbeing Taskforce (2015) report found waiting times of 32 weeks, with both referral rates and waiting times increasing, whilst Frith (2017) found waiting times of up to 38 weeks for assessment and 70 weeks to treatment. The most recent NHS Digital data found some improvements. The average waiting time in England between a referral and second contact for children and young people's mental health services for April 2019 to March 2020 was 43 days, although 6\% of CYP waited over 12 weeks (NHS Digital, 2020).

\section{Latest government policy and the emergence of new low-intensity psychological practitioner roles for CYP}

To address the current challenges in timely access to evidence-based psychological interventions for CYP experiencing mental health difficulties, recent government policy in England has included the development of new graduate psychological practitioner roles and training, and rapid expansion of this workforce. Primary aims of this are to increase capacity, and access in nonclinical settings, which have been identified as two of the key barriers (Reardon et al., 2019; House of Commons Health Committee, 2014). This policy centres on the new Children's Wellbeing Practitioner (CWP) and Education Mental Health Practitioner (EMHP) roles, both of which have a nationally developed 12-month training curricula (Department of Health and Social Care \& Department of Education, 2017, 2018; Department of Health CYP Mental Health \& Wellbeing Taskforce, 2015; Health Education England, 2017; Independent Mental Health Taskforce, 2016; NHS, 2019). Both programmes include core training in the delivery of low-intensity (single-strand, goal-focused, limited session) CB interventions for mild-moderate anxiety, low mood and behaviour difficulties, delivered via guided self-help, 1:1 interventions, group work and parent-led approaches. CWPs predominantly work in the NHS or voluntary/ community sector providers, whilst EMHPs are based in educational settings and work as part of newly established "Mental Health Support Teams" serving clusters of schools focusing on preventative work, early intervention, and whole school approaches to mental health and emotional wellbeing (for further details see Department of Health and Social Care \& Department of Health, 2017, 2018).

Expansion in the number of practitioners trained in these new roles is a substantial investment and it is therefore crucial to ensure that interventions delivered by this new workforce in the context of the training model are clinically effective and meaningful. We would argue that this is crucial to ensuring that what is being delivered by this workforce can truly be considered evidence-based. 


\section{Existing evidence on the effectiveness of $C B$ interventions delivered by graduate-level workers}

Prior to the emergence of the new roles outlined above, evidence on the effectiveness of $\mathrm{CB}$ interventions delivered to CYP by graduate-level workers was typically in the context of research trials evaluating the efficacy of a specific manualised approach for a specific presenting difficulty, and often delivered in groups (Cobham et al., 2017; Gallagher et al., 2004; Ginsburg and Drake, 2002; Holmes et al., 2014; Melfsen et al., 2011; Olivares-Olivares et al., 2019; Rapee et al., 2006a; Simon et al., 2011). In the UK, positive improvements have been demonstrated for CB interventions delivered to CYP by assistant/trainee psychologists and graduate-level workers for both anxiety (McConachie et al., 2014; Thirlwall et al., 2013) and depression (Arnott et al., 2020; Pass et al., 2018a; Pass et al., 2018b). One study directly compared experienced versus novice therapists in the delivery of $\mathrm{CB}$ interventions to CYP with anxiety, and found that CYP's outcomes were similar, regardless of the experience level of the therapist (Thirlwall et al., 2013). Other research is mixed as to what extent the experience level of the therapist has an impact on CYP outcomes following CB interventions (Bjaastad et al., 2018; Podell et al., 2013).

There is some preliminary emerging evidence from non-peer reviewed publications, suggesting that $\mathrm{CB}$ interventions delivered by practitioners in the new roles outlined above may be efficacious, with significant improvements in standardised outcome measures of symptom severity and symptom impact, and idiosyncratic goal measures, with medium to large effect sizes (Fuggle and Hepburn, 2019; Mavji et al., 2018). The initial report (Mavji et al., 2018) was an audit of the first cohort of the children's wellbeing practitioner programme (CWP), designed to evaluate the implementation of the programme across 15 London sites. The second report (Fuggle and Hepburn, 2019) is a summary of the clinical outcomes collated from the national CWP programme to update commissioners on the potential effectiveness of the programme. Initial findings from the CWP programme have suggested that the outcomes from this graduate workforce are comparable to those of the Children and Young People's Improving Access to Psychological Therapies project (CYP-IAPT) and the existing Child and Adolescent Mental Health Services (CAMHS) clinical workforce (Fuggle and Hepburn, 2019).

To the authors' knowledge, there have not been any other published peer-reviewed studies to date estimating the clinical effectiveness of CB interventions delivered to CYP by graduate-level practitioners or trainee graduate-level practitioners: (a) receiving training in a range of $\mathrm{CB}$ interventions for both mild to moderate anxiety and depression; or (b) working across community settings (NHS clinics and/or schools).

The aim of the current evaluation was therefore to build on the emerging evidence from Fuggle and Hepburn (2019) and Mavji et al. (2018) through conducting a practice-based evaluation to estimate the effectiveness of low-intensity CB interventions delivered by trainees on a one-year postgraduate course. As this was a routine practice-based evaluation, no control or comparison groups were available, and there are no long-term follow-up data.

\section{Method}

\section{Design}

This was a retrospective practice-based service evaluation into the outcomes of low-intensity CB interventions delivered by trainees from a single cohort of low-intensity psychological practitioners (CYP) during their training placements in CAMHS community clinics, or primary and secondary schools in the South East of England. CB interventions targeted (a) mild to moderate sleep difficulties; (b) poor eating patterns or diet; (c) low mood, depression and low self-esteem; or (d) anxiety difficulties including phobias, separation anxiety, social anxiety, school avoidance; and excessive worry. This evaluation focused on 
face-to-face 1:1 interventions with varying degrees of caregiver involvement, and did not include guided self-help, group, or parent-led CB interventions.

\section{Procedure}

The low-intensity CB interventions evaluated in this report were undertaken during trainees' clinical placements as part of the one-year Post-Graduate Certificate in Low-Intensity Psychological Interventions for CYP, run jointly by the University of Sussex and Sussex Partnership NHS Foundation Trust (SPFT). Trainees varied in age, academic background, and level of experience working with CYP and/or mental health difficulties. Key details of the training programme and trainee selection criteria are summarised in Box 1.

Box 1. Key details of training programme

\section{Programme structure}

Four 15-credit modules, each consisting of six teaching days and delivered consecutively*

- Developmental Models, Psychopathology, and Healthcare Legislation for Children and Young People

- Fundamentals of Therapeutic Interaction with Children and Young People

- Therapeutic Assessment, Engagement and Intervention with Children and Young People

- Cognitive Behavioural Therapy (CBT) Informed Interventions with Children and Young People

\section{Teaching approach}

Workshop based, utilising a range of methods including face-to-face teaching, facilitated web-based learning, clinical skills practice and experiential exercises, tutor-supported action learning groups, practice-based learning projects, and clinical supervision

Taught clinical/cognitive behavioural skills

Therapeutic relationship skills, assessment of a presenting problem, collaborative goal setting, risk screening, developing shared formulations using trans-diagnostic cognitive or behavioural theories of maintenance ( $A B C$ or cognitive triangle), and setback management

Cognitive behavioural interventions of health promotion (sleep hygiene and healthy eating), cognitive restructuring/change methods ${ }^{\star \star}$, graded exposure, worry tree, problem solving, and full behavioural activation for depression

\section{Assignments}

Combination of academic (essays, reflective reports) and clinical (clinical report, Objective Structured Clinical Examinations, recordings, portfolios) assignments. Trainees require 90 hours of direct cognitive behavioural assessment/interventions and at least six completed interventions

\section{Trainee selection}

Competitive selection process, including interview, screening for academic potential, clinical skills and knowledge, and therapeutic qualities (including interpersonal style, reflectiveness and resilience). Applicants required to hold an undergraduate degree at a UK 2:1 equivalent or higher, or to provide an academic portfolio evidencing capacity to study at postgraduate level. Psychology undergraduate degree is not a pre-requisite

* Module titles and weighting differ from those suggested in the national CWP and EMHP curricula, although the exit award, core learning objectives, and teaching content are the same.

${ }^{\star}$ Behaviourally based interventions for both anxiety and depression are mandatory content in the national curricula for CWP and EMHP training. Cognitive change methods are an optional component at the discretion of the University delivering the training. Given the evidence base for both cognitive and behavioural components of interventions (as noted in the introduction to this article), the training course undertaken by trainees in the current practice-based evaluation included two days of teaching on cognitive change methods as a core component of the training for all trainees. Cognitive change teaching covered three- and seven-column thought records, responsibility pie charts, thinking errors, and behavioural experiments. 
Clinical placements commenced three months after the course start date. During these first three months of the course, trainees were classroom-based, and took part in teaching and skills practice on $\mathrm{CB}$ assessment, formulation and intervention. Trainees were taught the following $\mathrm{CB}$ interventions: health promotion (healthy eating and sleep hygiene); graded exposure; cognitive restructuring; worry tree; problem solving; and behavioural activation. Trainees were required to pass a formal competency assessment in the form of an Objective Structured Clinical Examination (OSCE) prior to commencing direct work with CYP. Competency was assessed using the 12 domains on the Cognitive Therapy Scale-Revised (CTS-R; James et al., 2001) with a truncated scoring system and amended wording of some items to reflect low-intensity $\mathrm{CB}$ practice (available from the corresponding author on request). Trainee competence and fidelity to the models was assessed further throughout the clinical placement through an additional two OSCEs and two session recording submissions, all scored on the above competency scale. Trainees received weekly group supervision from a British Association of Behavioural and Cognitive Psychotherapists (BABCP) accredited CBT therapist with experience of working with CYP.

CYP were typically seen for two assessment sessions to gain information regarding the presenting difficulty, wider contextual influences, severity, risk and goals. Routine outcome measures (ROMS) were completed either within assessment sessions or between the first and second session. Assessment included discussion with caregivers for all CYP of primary school age (up to 11 years old), and for CYP of secondary school age who gave consent for this. Information from each assessment was discussed with the supervisor and it was agreed whether suitability for a low-intensity CB intervention was met. Suitability was determined based on a combination of generic CB suitability criteria (Safran and Segal, 1990; Stallard, 2002) and the presenting difficulty meeting the criteria of being (a) covered within the taught course programme (see Box 1 for more details); (b) mild to moderate in severity; (c) low complexity; (d) low risk; and (e) the CYP had an absence of an autism or learning disability diagnosis.

Where the suitability criteria were met, it was determined through supervision whether a cognitive or behavioural intervention was indicated, based on key maintaining factors, developmental level, and the evidence base for the specific presenting difficulty. An individualised maintenance formulation was developed with the CYP in the following session using either a behavioural ABC (Kuyken et al., 2011; Richards and McDonald, 1990) or cognitive triangle (Greenberger and Padesky, 2016) model, and the rationale and nature of the recommended intervention was discussed. For behavioural activation, the Martell et al. (2001) formulation model was used. Where more than one intervention could have been beneficial, CYP were provided with the rationale and details of each and selected their preferred option. If the CYP (and caregivers for those of primary school age) consented to the intervention, weekly sessions followed, utilising key principles, steps and suggested sequencing of the chosen intervention (note that interventions were not manualised but did follow a protocol). Interventions included some form of caregiver involvement for all primary school aged CYP and for secondary school aged CYP who consented to their caregiver's involvement. ROMS were repeated at the end of the intervention and represented routine service data collection. The final intervention session with CYP covered lapse prevention.

\section{Participants}

The suitability criteria described above was shared with training placements, who referred CYP aged between 6 and 18 years experiencing sleep difficulties, poor eating patterns or diet, low mood, or anxiety-based difficulties, to the trainees. An assessment was offered if the CYP's perspective was included in the referral, the CYP (and caregivers for primary school aged CYP) consented, and there were no clear indications from the referral that the CYP did not meet the above 
suitability criteria. CYP already receiving an ongoing 1:1 psychological intervention for the same presenting difficulty they were referred to the trainee for were not offered an assessment.

\section{Measures}

All measures used in the current practice-based evaluation are part of the routine data set agreed as part of the national CYP Improving Access to Psychological Therapies (CYP-IAPT) initiative in England (see Scott, 2018), and the standardised measures have established validity and reliability. Baseline measures were sought and reviewed as part of the assessment process and repeated at the end of interventions. Measures were completed either within the sessions, or set as homework to be completed in between sessions.

\section{Strengths \& Difficulties Questionnaire (SDQ) (Goodman, 2001)}

CYP aged 11-17 years old were asked to self-complete the child SDQ, whilst caregivers of CYP aged between 6 and 17 years were asked to complete the parent SDQ. The SDQ is a 25-item questionnaire and is divided into five subscales (emotional symptoms, conduct problems, hyperactivity-inattention, peer problems, and prosocial behaviour). Questions are rated using a 3-point Likert scale (not true, somewhat true, and certainly true). A total difficulties score is generated by summing the four subscales of emotion, conduct, hyperactivity-inattention, and peer problems, with a maximum score of 40 . Items relating to pro-social behaviours are not included in the total difficulties score. A 4-fold cut-point classification has been created where a CYP's self-reported total difficulties score is grouped as either close to average (0-14), slightly raised (15-17), high (18-19), or very high (20-40). A similar 4-fold cut-point classification has been created for the parent-report total difficulties score, grouped as either close to average (0-13), slightly raised (14-16), high (17-19), or very high (20-40). For the SDQ total difficulties, clinical thresholds are defined as those scoring 15 or more on the selfreport measure, and 14 or more on the parent-reported measure.

CYP and caregivers were also asked to complete the SDQ impact supplement. The SDQ impact supplement is scored by measurement of five items on distress and impairment which are summed to generate a total impact score. There is a 4-point Likert response (not at all, only a little, a medium amount, and a great deal) with scores ranging from 0 to 10 , and higher scores indicating greater impact. A 4-fold cut-point classification has been created where the total impact score is grouped as either close to average (0), slightly raised (1), high (2), or very high (3-10). For the SDQ impact supplement, clinical thresholds are defined as those scoring 1 or more on the impact scale.

The teacher SDQ measure was available for trainees to use; however, its use was optional given that not all placements were in educational settings, and depended on the CYPs' consent to seek information from teachers. Teacher-reported SDQ scores are not reported in the current-practice based evaluation as insufficient data were available.

\section{Revised Children's Anxiety and Depression Scale (RCADS) (Chorpita et al., 2000)}

All CYP and their caregivers were asked to complete either the Revised Children's Anxiety and Depression Scale (RCADS), or the RCADS Parent Version (RCADS-P), respectively. The RCADS is a 47 -item questionnaire that measures the frequency of various symptoms of anxiety and low mood to produce a total anxiety and depression combined score and separate scores for each of the follow sub-scales: separation anxiety; social phobia; generalized anxiety; panic; obsessive compulsive; total anxiety; total depression. Responses are provided on a 4-point Likert scale (0, never; 3, always) where higher scores indicate greater difficulties. $t$-scores are then calculated from the raw scores using the spreadsheet, or $t$-score conversion tables, provided by the developers (Chorpita et al., 2000). For the RCADS, clinical thresholds are defined as those 
Table 1. Client demographics

\begin{tabular}{ll}
\hline Characteristic & $N=109$ \\
\hline Gender & Female: $n=64(58.7 \%)$ \\
Age & Male: $n=45(41.3 \%)$ \\
& Mean $=12.17$ years $(S D=3.23)$, range $6-17$ years \\
Placement type & $\leq 11$ years old: $n=49(45 \%)$ \\
& $\geq 12$ years old: $n=60(55 \%)$ \\
Number of sessions & Schools: $n=62(56.9 \%)$ \\
& CAMHS: $n=46(42.2 \%)$ \\
& $8.76(4.37)$ range $1-20$ \\
& Schools: $9.21(4.25)$ range $1-20$ \\
& CAMHS: mean $=8.20(S D=4.55)$ range $1-20$ \\
\hline
\end{tabular}

${ }^{\mathrm{a}} N=108 .{ }^{\mathrm{b}} \mathrm{N}=106$.

with a $t$-score of 70 or higher. Children aged $<8$ years were removed from the RCADS analysis as there are no norms for this age group.

\section{Goal-based outcomes (GBO) (Law and Wolpert, 2014)}

During the assessment, all CYP were asked to set themselves at least one SMART (Specific, Measurable, Attainable, Realistic, and Timely) goal (maximum of three), which they would like to achieve by the end of the intervention. CYP were asked to rate their goals from 0 (no progress made towards their goal) to 10 (the goal has been met). For the purposes of analysis, only the primary goal has been included due to variation in the number of goals set by CYP.

\section{Analysis}

Data were first inspected to check all the assumptions associated with parametric tests were met. Any issues with bias were corrected using either a transformation or bootstrapping. Independent $t$-tests were used to compare baseline differences between CAMHS and school placements to detect any differences in severity. Paired samples $t$-tests were used to detect the difference between pre- and post-intervention scores on CYP outcomes for (a) the SDQ total difficulties, and total impact scores; (b) the RCADS total anxiety, and total depression subscales, as well as the total anxiety and depression score; and (c) the primary GBO score. Cohen's $d$ was calculated to determine the effect size of these differences, with effect sizes defined as small (0.3), medium (0.5) and large (0.7) (Cohen, 1988). Chi-squared analyses were conducted to detect differences in the number of CYP over the clinical cut-off on measures pre- and postintervention. Missing data were not replaced, and cases were excluded on a case-by-case basis.

The CYP were categorised into those with complete or incomplete data. Those with complete data were defined as having paired data for at least one outcome measure.

\section{Results}

\section{Demographics, and clinical and intervention characteristics}

Demographic data were collected for 109 CYP. There was a relatively even split between males/ females and school/CAMHS placements in the current practice-based evaluation (see Table 1). Recipients of interventions represented the full age range likely to receive $\mathrm{CB}$ interventions in routine practice, with a mean age of 12 years. CYP were categorised into two age brackets: younger CYP ( $\leq 11$ years old) and older CYP ( $\geq 12$ years old), and found they were similarly represented in our sample, $45 \%$ and $55 \%$, respectively. The frequency of different interventions being delivered in the current practice-based evaluation are reported (Fig. 1), 


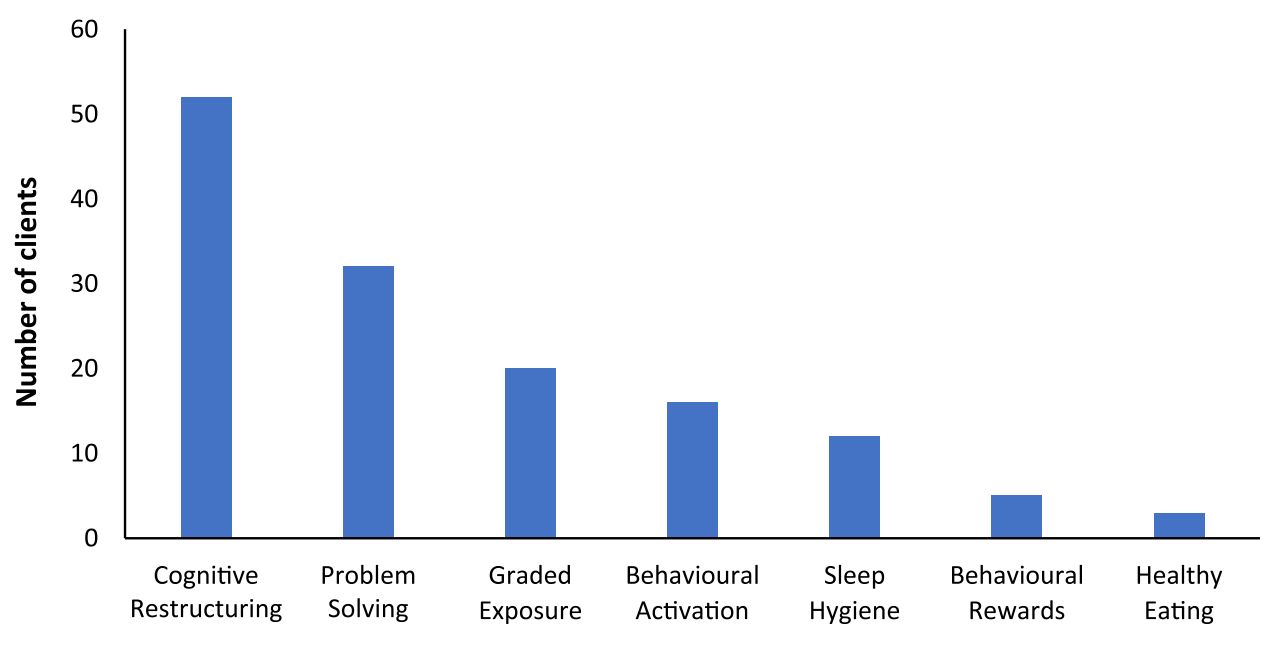

Interventions delivered

Figure 1. Interventions delivered.

with interventions that can be utilised for both anxiety and depression most frequently utilised (cognitive restructuring and problem solving). The average number of sessions delivered was similar across both schools and CAMHS placements, with nine and eight sessions, respectively. A small proportion of CYP (9.2\%) were receiving CAMHS treatment for other presenting difficulties at the same time as the trainee-delivered interventions.

There were differences in baseline symptom severity between those CYP seen in CAMHS clinics and those seen in a school setting (see Table 2). Significant differences were found across several of the core measures, with CYP in CAMHS often experiencing higher levels of symptoms and impact scores than those seen in school settings. For example, the mean SDQ total difficulties scale score fell in the high category for those CYP seen in CAMHS, and in the slightly raised category for those seen in schools.

On the data available (both parent and CYP reported RCADS), there were no significant differences in baseline measures between those with complete data, and those with incomplete data (all $p>.05$ ). There were no significant differences between those with complete and incomplete data by age, gender, or placement type.

\section{Clinical outcomes - self-reported and parent measures}

Scores on all self- and parent-rated measures pre- and post-intervention, irrespective of the placement setting or intervention delivered, are presented in Table 3. Scores for the overall sample are presented, as the sample size prevented sufficiently powered statistical analysis by sub-group. Significant improvements were found across all core outcome measures, including symptom severity, impact of the difficulties, and CYP goals (all $p<.05$ ). Improvements in CYP self-rated scores across the standardised questionnaires all demonstrated medium effect sizes (Cohen's $d=.53-.62$ ), whilst self-rated scores on the idiosyncratic goals-based outcome measure showed a large effect size (Cohen's $d=2.57$ ). Parent-rated measures also demonstrated small to large effects sizes across the standardised questionnaires (Cohen's $d=.32-.89$ ).

Change in participants meeting clinical thresholds for all symptom outcome measures were examined using chi-squared analysis. At baseline between 30 and $45 \%$ of the sample (CYP and parent) reported difficulties which would indicate meeting the clinical threshold. After 
Table 2. Placement type baseline differences

\begin{tabular}{lrrr}
\hline & \multicolumn{2}{c}{ Mean (SD) } \\
Outcome ( $n$ CAMHS, $n$ Schools) & \multicolumn{1}{c}{ CAMHS } & Schools & t-statistic \\
\hline Self-reported measures & & & -1.38 \\
CYP SDQ Total difficulties (35, 27) & $19.20(5.65)$ & $17.04(6.66)$ & $-4.03^{\star \star \star}$ \\
CYP SDQ Total impact (32, 22) & $5.63(2.55)$ & $2.82(2.46)$ & $-4.31^{\star \star \star}$ \\
CYP RCADS Anxiety (42, 43) & $69.43(16.63)$ & $53.93(16.49)$ & $-4.56^{\star \star \star}$ \\
CYP RCADS Depression (42, 41) & $71.43(17.95)$ & $54.41(15.95)$ & $-4.33^{\star \star \star}$ \\
CYP RCADS Anxiety and Depression (42, 41) & $70.50(17.32$ & $54.15(17.08)$ & .38 \\
Parent-reported measures & & & $-3.20(7.60)$ \\
Parent SDQ Total difficulties (25, 29) & $16.45(6.99)$ & $3.54(2.99)$ & $-2.40^{\star}$ \\
Parent SDQ Total impact (28, 24) & $5.502 .87)$ & $67.59(16.24)$ & $-2.17^{\star}$ \\
Parent CYP RCADS Anxiety (30, 22) & $77.97(18.29)$ & $68.73(14.04)$ & -1.21 \\
Parent RCADS Depression (30, 22) & $74.60(19.22)$ & $68.65(16.25)$ & $-2.49^{\star}$ \\
Parent RCADS Anxiety and Depression (30, 22) & $80.53(16.81)$ & & \\
\hline
\end{tabular}

${ }^{\star * \star} p<.001,{ }^{\star} p<.05$, children aged $<8$ years were removed from the RCADS analysis.

Table 3. CYP and parent-rated outcome measures

\begin{tabular}{|c|c|c|c|c|c|c|}
\hline \multirow[b]{2}{*}{ Outcome } & \multirow[b]{2}{*}{$n$} & \multicolumn{2}{|c|}{ Mean $(S D)$} & \multirow[b]{2}{*}{ T-value } & \multirow[b]{2}{*}{ BCa bootstrapped $95 \% \mathrm{Cl}$} & \multirow{2}{*}{$\frac{\text { Effect size }}{\text { Cohen's } d}$} \\
\hline & & Pre & Post & & & \\
\hline \multicolumn{7}{|l|}{ Self-reported measures } \\
\hline SDQ Total difficulties & 47 & $18.83(6.63)$ & $14.68(6.06)$ & $7.08^{\star \star \star}$ & [3.12-5.15] & .62 \\
\hline SDQ Total impact & 39 & $4.69(2.61)$ & $3.21(2.59)$ & $4.42^{\star \star \star}$ & {$[.82-2.10]$} & .58 \\
\hline RCADS Anxiety & 65 & $61.83(17.99)$ & $52.51(15.18)$ & $6.89^{\star \star \star}$ & {$[6.79-11.93]$} & .56 \\
\hline RCADS Depression & 63 & $62.43(18.18)$ & $53.33(16.31)$ & $5.70^{\star \star \star}$ & {$[5.91-12.60]$} & .53 \\
\hline RCADS Anxiety and Depression & 62 & $63.06(18.42)$ & $52.48(15.52)$ & $6.89^{\star \star \star}$ & {$[7.50-13.63]$} & .62 \\
\hline Primary goals & 66 & $2.14(2.00)$ & $7.95(2.52)$ & $-18.04^{\star \star \star}$ & {$[-6.52-5.20]$} & 2.57 \\
\hline \multicolumn{7}{|l|}{ Parent-reported measures } \\
\hline SDQ Total difficulties & 31 & $17.55(8.11)$ & $15.16(7.09)$ & $2.02^{\star}$ & {$[.13-4.65]$} & .32 \\
\hline SDQ Total impact & 30 & $5.33(2.82)$ & $2.90(2.75)$ & $4.46^{\star \star \star}$ & {$[1.40-3.47]$} & .89 \\
\hline RCADS Anxiety & 32 & $75.13(16.60)$ & $68.59(15.07)$ & $3.40^{\star \star}$ & [3.19-9.68] & .41 \\
\hline RCADS Depression & 32 & $72.91(18.98)$ & $66.34(15.48)$ & $2.04^{\star}$ & {$[0.03-12.12]$} & .38 \\
\hline RCADS Anxiety and Depression & 32 & $77.69(16.10)$ & $68.81(14.33)$ & $3.99^{\star \star \star}$ & {$[4.71-13.57]$} & .58 \\
\hline
\end{tabular}

${ }^{* \star *} p<.001,{ }^{* *} p<.01,{ }^{*} p<.05$, children aged $<8$ years were removed from the RCADS analysis.

completion of the CB interventions a significant reduction was observed in all CYP and parentreported outcomes, with those meeting thresholds decreasing to 7-30\% (Table 4).

\section{Discussion}

Recent NHS data suggest that the prevalence of mental health disorders is rising in CYP in England (Sadler et al., 2018; Vizard et al., 2020), and any additional effects of the current worldwide pandemic are as yet unknown. Anxiety and depression are amongst the most common mental health difficulties experienced by CYP and effective psychological interventions exist. Timely access to these evidence-based psychological interventions has remained a significant challenge. Recent government policy in England has therefore sought to increase early access through training a non-traditional graduate workforce to deliver a range of low-intensity CB interventions to CYP in a variety of settings (Department of Health and Social Care \& Department of Education, 2017; Health Education England, 2017). To our knowledge this is one of the first peer-reviewed articles to estimate the effectiveness of CB interventions for CYP: (i) delivered by graduates from a range of backgrounds; (ii) delivered 
Table 4. Clinical threshold change on outcome measures

\begin{tabular}{|c|c|c|c|}
\hline Outcome & $\begin{array}{l}\text { CYP meeting clinical threshold at } \\
\text { baseline, } n(\%)\end{array}$ & $\begin{array}{l}\text { CYP meeting clinical threshold post CB } \\
\text { intervention, } n(\%)\end{array}$ & $\begin{array}{l}\text { Chi-squared } \\
\text { statistic }\end{array}$ \\
\hline \multicolumn{4}{|c|}{ Self-reported measures } \\
\hline SDQ Total difficulties & $49(45.0)$ & $28(25.7)$ & $46.14^{\star \star \star}$ \\
\hline SDQ Total impact & $48(44.0)$ & $32(29.4)$ & $45.23^{\star \star \star}$ \\
\hline RCADS Anxiety & $31(30.7)$ & $8(7.9)$ & $32.87^{\star \star \star}$ \\
\hline RCADS Depression & $32(29.4)$ & $8(7.3)$ & $25.95^{\star \star \star}$ \\
\hline $\begin{array}{l}\text { RCADS Anxiety and } \\
\text { Depression }\end{array}$ & $30(30.0)$ & $8(8.0)$ & $31.82^{\star \star \star}$ \\
\hline \multicolumn{4}{|c|}{ Parent-reported measures } \\
\hline SDQ Total difficulties & $35(32.1)$ & $17(15.6)$ & $29.10^{\star \star \star}$ \\
\hline SDQ Total impact & $45(41.3)$ & $23(21.1)$ & $30.09^{\star \star \star}$ \\
\hline RCADS Anxiety & $32(31.7)$ & $15(14.9)$ & $48.67^{\star \star \star}$ \\
\hline RCADS Depression & $30(29.7)$ & $15(14.9)$ & $30.93^{\star \star \star}$ \\
\hline $\begin{array}{l}\text { RCADS Anxiety and } \\
\text { Depression }\end{array}$ & $34(33.7)$ & $16(15.8)$ & $43.69^{\star \star \star}$ \\
\hline
\end{tabular}

${ }^{\star \star *} p<.001$, children aged $<8$ years were removed from the RCADS analysis

whilst graduates were still in training; (iii) where graduates were trained in a range of $\mathrm{CB}$ interventions rather than one specific protocol/manualised approach; (iv) delivered for a range of low-mood or anxiety-based problems; and (v) delivered in community settings (both healthcare clinics and schools).

Results of the current practice-based evaluation are encouraging in showing positive and meaningful improvements across a range of mild to moderate low-mood and anxiety-based difficulties following a range of low-intensity $\mathrm{CB}$ interventions, delivered by trainee postgraduate practitioners from a range of backgrounds, and delivered in either schools or community CAMHS clinics. Mean scores reduced for the whole sample, representing significant improvements across standardised self- and parent-rated measures of symptom severity, impact, and self-reported idiosyncratic goal measures. Effect sizes were predominantly medium to large (Cohen, 1988). The number of CYP over clinical thresholds on standardised measures significantly reduced post-intervention. The reductions in both mean scores and the proportion of CYP meeting clinical thresholds on the SDQ may suggest wider improvements beyond anxiety and low mood symptoms. Despite less data, the most significant improvements were on parental reports regarding the impact of difficulties on daily life. This is notable given that direct caregiver involvement in interventions varied, so caregiver feedback was arguably a more objective source of feedback, although equally they may not have a full and accurate insight into their child's emotional state. Findings from this practice-based evaluation are also notable given that they were achieved whilst practitioners were still in training. Within the current practice-based evaluation the majority of CYP received cognitive restructuring, followed by problem solving. This would be expected given that cognitive restructuring and problem solving can be utilised for CYP with both low-mood and anxiety-based difficulties (e.g. Cool Kids/Chilled Anxiety and Depression Programmes; Rapee et al., 2006b), whilst other interventions covered in the training programme were more specific to one type of difficulty (e.g. behavioural activation for depression).

Given the unique aims of this practice-based evaluation, direct comparison with previous research is difficult. The closest comparisons are with the national CWP programme outcomes evaluation by Fuggle and Hepburn (2019), and the London and South East England CWP audit report by Mavji et al. (2018), both of which also found significant improvements across the same standardised measures and idiosyncratic goal measures used in the current evaluation. Similar to Fuggle and Hepburn (2019), the current practice-based evaluation found recovery rates were higher for the anxiety and depression outcome measure (RCADS) 
compared with the SDQ measure. This may be anticipated as the interventions delivered specifically targeted anxiety and/or depression symptoms, as measured by the RCADS, whilst the SDQ measures a wider set of mental health symptoms. Effect sizes in these CWP reports were slightly larger for standardised measures and lower for idiosyncratic goals-based measures than the current evaluation, although both of these previous reports included various modes of $\mathrm{CB}$ delivery (guided self-help, group-work, parent-led), data from qualified and trainee CWPs, and only utilised parent-rated SDQ for primary school age children and self-report RCADS for secondary school age young people, so are not entirely comparable.

The findings from the current practice-based evaluation expand upon the existing evidence base for the training of graduate-level practitioners to deliver $\mathrm{CB}$ interventions to CYP. In the UK, Arnott et al. (2020) reported medium effect sizes following a 1:1 behavioural activation intervention for overweight and obese adolescents with low mood delivered by a graduate therapist in a community setting. Pass et al. (2018b) also found large effect sizes for behavioural activation delivered in a CAMHS setting by graduate psychologists. Both Arnott et al. (2020) and Pass et al. (2018b)'s effect sizes were calculated with small sample sizes of eight and $20 \mathrm{CYP}$, respectively, and Arnott et al. also used different outcome measures to assess low mood to that of the current practice-based evaluation. Similar estimations of effect sizes were found in the current practice-based evaluation, with effect sizes predominantly medium to large for self-rated outcome measures. This is promising considering that for the current practice-based evaluation all trainees took part in the same classroom-based training regardless of placement type, and delivered a range of CB interventions, demonstrating similar effect sizes to the above studies specialised to a specific setting and one specific manualised behavioural activation protocol. Regarding clinical thresholds, Thirlwall et al. (2013) found that up to $50 \%$ of CYP recovered from their primary anxiety disorder following a guided CB intervention delivered by a graduate-level practitioner. In the current practice-based evaluation, the number of CYP meeting the clinical threshold across the parent-reported outcome measures approximately halved following $\mathrm{CB}$ interventions. This is significant given that the current findings are based on information collected from this graduate-level workforce whilst still in training, and there was no pre-requisite for trainees to have an undergraduate degree in a psychology-related discipline. In comparison, Thirlwall et al.'s novice graduate-level practitioners were either assistant psychologists, or psychology graduates. The findings in the current practice-based evaluation are also consistent with previous research that has found similar reductions in the percentage of CYP meeting the clinical threshold following a group CB intervention delivered by graduate-level workers (Cobham et al., 2017; Gallagher et al., 2004; Holmes et al., 2014; Simon et al., 2011). All four of these studies used different outcome measures compared with the current practice-based evaluation, and the sample sizes were often small, with three of the studies each delivering a CB intervention to less than 35 CYP (Cobham et al., 2017; Gallagher et al., 2004; Holmes et al., 2014). There were also large variations in the duration of the intervention delivered, with interventions ranging from three (Gallagher et al., 2004) to 10 sessions (Holmes et al., 2014). Although the authors recognise that it is difficult to make conclusive direct comparisons with previous clinical research due to differences in settings, the severity of the presenting problems, the intervention protocols, and the outcome measures used, our findings are generally supportive of previous clinical research. We acknowledge there is a need for robust research to be conducted regarding the training of graduates to deliver 1:1 $\mathrm{CB}$ interventions before strong conclusions can be drawn.

Further comparisons with previous wider research into CB interventions with CYP would be problematic given that these were often undertaken in controlled research settings, with manualised protocols for one specific type of problem, and often delivered by experienced practitioners or therapists (Ewing et al., 2015). However, results of the current evaluation show some equivalence with prior studies of CYP symptomatic of depression referred and 
seen within the community or their schools (e.g. Calear and Christensen, 2010; Weisz et al., 2006). Whilst recent meta-analyses have shown mixed findings on the effectiveness of CB interventions for mild or sub-clinical anxiety and depression (Asarnow et al., 2001; Caldwell et al., 2019; Johnstone et al., 2018; Rasing et al., 2017; Stockings et al., 2016; Werner-Seidler et al., 2017), results of this practice-based evaluation support the potential effectiveness, as the sample included CYP with mild or sub-clinical anxiety and depression.

There were several strengths in the current practice-based evaluation. The use of standardised and idiosyncratic measures and of both self and parent-report strengthened confidence in the findings. Measures captured a range of outcomes including overall mental health symptom severity, anxiety and depression symptom severity, impact of symptoms, and idiosyncratic goal progress.

The sample represented a relatively even split between males and females, and the full range of ages that CB interventions are likely to be targeted at in CYP. This is relatively unique in comparison with previous studies where a specific age group is often targeted and/or community-based studies that can often unintentionally have a higher percentage of female participants (e.g. Pass et al., 2018a,b,c).

The current practice-based evaluation also had a relatively even split between CAMHS and school-based placements. There were some significant differences between placements in terms of severity and impact of initial presenting difficulties, with higher severity and impact in CYP within CAMHS. However, this would be expected in terms of CAMHS thresholds, and is consistent with the principle of targeting earlier intervention in non-CAMHS settings. Additionally, as mean scores and the number of CYP over clinical cut-offs reduced significantly for the overall sample irrespective of placement, these findings would suggest that CB interventions delivered by graduate-level workers have the potential to be effective for differing levels of severity and when delivered in both healthcare clinics and school settings.

Despite the encouraging findings, limitations of the current practice-based evaluation should be noted. The size of the data set did not allow for any meaningful or reliable comparisons of outcomes between different interventions, intervention duration, placement settings, gender or ages. Data on a range of other demographic characteristics such as ethnicity, family composition, and socio-economic status were not collated and it is therefore not possible to state how representative the current sample was. This is a key area for future research. Neither was information available on whether CYP were receiving any concurrent mental health support, although trainees did not provide interventions to CYP receiving other ongoing 1:1 psychological interventions for the same difficulty. The present results are confined to interventions delivered in a 1:1 format, whereas aspirations for this practitioner role moving forwards include guided self-help and group work, so further research is needed into the efficacy of these other modes of delivery by graduate-level workers. The process of completing outcome measures was also not standardised, with trainees administering these measures to CYPs and parents in a way that suited the individual client (i.e. whether during a session, or as homework). We therefore do not know the extent to which any of these selfreport measures were completed by parents acting as a proxy. Lastly, given that this report represented a routine practice-based evaluation, no control or comparison groups were available, and there is no longer-term follow-up data on the sustainability of improvements.

There are a number of potential research, policy and practice implications from the current evaluation. It is critical that further robust studies are conducted as this new workforce is expanded. These should include larger, representative samples to enable comparison of effectiveness between the different settings CB interventions are delivered in, mode of delivery (e.g. 1:1, guided self-help, group work, parent-led), specific intervention techniques, and any differences based on key demographic characteristics of the CYP. Inclusion of control or comparison groups, and long-term follow-up on outcomes, will be important to establish both the relative effectiveness of $\mathrm{CB}$ interventions over no intervention or 'support as usual' and 
the sustainability of any gains. It is of interest that cognitive change was the most commonly utilised intervention in the current evaluation as this is not a mandatory component of the 1:1 interventions teaching content (see Box 1) within the national curriculum for these new lowintensity psychological practitioner roles in CYP mental health. The level of utilisation of cognitive change in the current evaluation may reflect its applicability across a range of lowmood, low self-esteem and anxiety-based difficulties, whereas other interventions are specific to one type of difficulty (e.g. behavioural activation for depression, worry tree for worry, graded exposure for phobic anxiety). Further evaluation into the specific effectiveness of cognitive change will help establish whether this particular intervention strategy is important to incorporate as an essential component of training for this new workforce.

In conclusion, results of the current practice-based evaluation are promising in terms of the capacity to train graduates from a range of backgrounds to deliver potentially effective $\mathrm{CB}$ interventions in CAMHS or school settings, to CYP of various ages experiencing a range of anxiety or low-mood based difficulties of varying severity. This practice-based evaluation provides some empirical evidence to support a postgraduate training model for increasing this non-traditional workforce. Further research with representative samples is needed into the long-term sustainability of improvements and to provide more detailed comparisons of outcomes across different settings, different modes of intervention delivery, and different interventions. Information on the desirability and satisfaction that CYP and families experience following interventions delivered by this new workforce is also crucial, as this is likely to have a significant impact on accessing interventions and attrition rates. Service recipient feedback is also a key principle of the CYP-IAPT programme (Scott, 2018). Qualitative feedback from the current practice-based evaluation sample is available and is currently being prepared for a sister article.

Key practice points

(1) Low-intensity cognitive and behavioural interventions delivered by trainee graduate-level practitioners to children and young people in schools and community mental health clinics were estimated to be effective in: improving mental health, anxiety and depression symptom severity and impact; reducing numbers of children and young people above clinical cut-offs on measures; and achieving personalised goals.

(2) The sample included children and young people experiencing mild and sub-clinical depression and anxiety.

(3) Interventions showed the potential to prove effective in the context of a 12-month training programme following national curricula in England for Children's Wellbeing Practitioners and Education Mental Health Practitioners, including regular competency assessment and weekly supervision.

(4) Cognitive restructuring/change methods were the most frequently utilised intervention but are not currently a mandatory component of the national curricula.

(5) Further robust evaluation of the effectiveness of the full range of interventions delivered by the new graduate-level low-intensity psychological practitioner roles in England is critical.

Acknowledgements. With thanks to the following practitioners for their support in collating data and delivering the interventions reported: Paola Furno, Emily Gribius, Lauren Heritage, Ruta Kackauskaite, Deborah Miller, Caroline Pearce, Beth Reader, Victoria Ripley, Shay Rosenthal, Vanessa Summers, Nicola Vince and Rosie Wooldridge. The authors would also like to thank Mary John and Petar Stermsek for their research advice and intern support.

Financial support. This research received no specific grant from any funding agency, commercial or not-for-profit sectors.

Conflicts of interest. The authors have no conflicts of interest with respect to this publication.

Ethical statements. The authors have abided by the Ethical Principles of Psychologists and Code of Conduct as set out by the British Association for Behavioural and Cognitive Psychotherapies (BABCP) and British Psychological Society (BPS). Following consultation with SPFT research and audit departments it was advised that ethical approval was not required for this report as it constituted service evaluation.

Data availability statement. As this was a practice-based evaluation of outcomes collected as part of routine practice, the data that support the findings of this study are not publicly available. 
Author contributions. Gavin Lockhart: Conceptualization, Data curation, Investigation, Methodology, Project administration, Resources, Supervision, Validation, Visualization, Writing-original draft, Writing-review \& editing; Christina Jones: Data curation, Formal analysis, Investigation, Methodology, Resources, Software, Writing-original draft, Writing-review \& editing; Victoria Sopp: Data curation, Formal analysis, Writing-review \& editing.

\section{Further reading}

Caldwell, D. M., Davies, S. R., Hetrick, S. E., Palmer, J. C., Caro, P., López-López, J. A., Gunnell, D., Kidger, J., Thomas, J., French, C., Stockings, E., Campbell, R., \& Welton, N. J. (2019). School-based interventions to prevent anxiety and depression in children andyoung people: a systematic review and network meta-analysis. The Lancet Psychiatry, 6, 1011-1020. https://doi.org/10.1016/S2215-0366(19)30403-1

Fuggle, P., \& Hepburn, C. (2019). Clinical outcomes for the wellbeing practitioner programme for children, young people, and their parents/ carers: Update report December 2019. Anna Freud National Centre for Children and Families. https:// manuals.annafreud.org/cwp/\#National\%20CWP\%20Outcomes\%20Report\%202019

Health Education England (2017). Stepping forward to 2020/2021. The mental health workforce plan for England. NHS England. https://www.hee.nhs.uk/sites/default/files/documents/Stepping\%20forward\%20to\%20202021\%20-\%20The\% 20 mental\%20health\%20workforce\%20plan\%20for\%20england.pdf

Johnstone, K. M., Kemps, E., \& Chen, J. (2018). A meta-analysis of universal school-based prevention programs for anxiety and Depression in children. Clinical Child and Family Psychology Review, 21, 466-481. https://doi.org/10.1007/s10567-0180266-5

National Health Service (2019). The NHS long term plan. https://www.longtermplan.nhs.uk

Stockings, E., Degenhardt, L., Dobbins, T., Lee, Y., Erskine, H., Whiteford, H., \& Patton, G. (2016). Preventing depression and anxiety in young people: A review of the joint efficacy of universal, selective and indicated prevention. Psychological Medicine, 46, 11-26. http://doi.org/10.1017/S0033291715001725

\section{References}

Andrews, G., Szabo, M., \& Burns, J. (2002). Preventing major depression in young people. British Journal of Psychiatry, 181, 460-462. https://doi.org/10.1192/bjp.181.6.460

Arnott, B., Kitchen, C., Ekers, D., Gega, L., \& Tiffin, P. A. (2020). Behavioural activation for overweight and obese adolescents with low mood delivered in a community setting: feasibility study. BMJ Paediatrics Open, 4, e000624. https://doi.org/10.1136/bmjpo-2019-000624

Asarnow, J. R., Jaycox, L. H., \& Tompson, M.C. (2001). Depression in youth: psychosocial interventions. Journal of Clinical Child \& Adolescent Psychology, 30, 33-47. https://doi.org/10.1207/S15374424JCCP3001_5

Banks. J., \& Xu, X. (2020). The mental health effects of the first two months of lockdown and social distancing during the Covid19 pandemic in the UK: IFS Working Paper W20/16. Institute for Fiscal Studies. https://www.ifs.org.uk/uploads/WP202016Covid-and-mental-health.pdf

Bignardi, G., Dalmaijer, E. S., Anwyl-Irvine, A. L., Smith, T. A., Siugzdaite, R., Uh, S., \& Astle, D. E. (2020). Longitudinal increases in childhood depression symptoms during the COVID-19 lockdown. Archives of Disease in Childhood. http://dx. doi.org/10.1136/archdischild-2020-320372

Bjaastad, J. F., Henningsen Wergeland, G. J., Mowatt Haugland, B. S., Gjestad, R., Havik, O. E., Heiervang, E. R., \& Öst, L. G. (2018). Do clinical experience, formal cognitive behavioural therapy training, adherence, and competence predict outcome in cognitive behavioural therapy for anxiety disorders in youth? Clinical Psychology \& Psychotherapy, 25, 865-877. https://doi.org/10.1002/cpp.2321

Brett, S., Reynolds, S., Totman, J., \& Pass, L. (2020). Brief behavioural activation therapy for adolescent depression in schools: two case examples. Emotional and Behavioural Difficulties, 25, 291-303. http://doi.org/10.1080/13632752.2020. 1861853

Caldwell, D. M., Davies, S. R., Hetrick, S. E., Palmer, J. C., Caro, P., López-López, J. A., Gunnell, D., Kidger, J., Thomas, J., French, C., Stockings, E., Campbell, R., \& Welton, N. J. (2019). School-based interventions to prevent anxiety and depression in children and young people: a systematic review and network meta-analysis. The Lancet Psychiatry, 6, 1011-1020. https://doi.org/10.1016/S2215-0366(19)30403-1

Calear, A. L., \& Christensen, H. (2010). Systematic review of school-based prevention and early intervention programs for depression. Journal of Adolescence, 33, 429-438. http://doi.org/10.1016/j.adolescence.2009.07.004

Care Quality Commission (2018). Are we listening? Review of children and young people's mental health services. https://www. cqc.org.uk/sites/default/files/20180308b_arewelistening_report.pdf

Cartwright-Hatton, S., Roberts, C., Chitsabesan, P., Fothergill, C., \& Harrington, R. (2004). Systematic review of the efficacy of cognitive behaviour therapies for childhood and adolescent anxiety disorders. British Journal of Clinical Psychology, 43, 421-436. https://doi.org/10.1348/0144665042388928 
Chorpita, B. F., Yim, L. M., Moffitt, C. E., Umemoto L. A., \& Francis, S. E. (2000). Assessment of symptoms of DSM-IV anxiety and depression in children: a Revised Child Anxiety and Depression Scale. Behaviour Research and Therapy, 38, 835-855. https://doi.org/10.1016/S0005-7967(99)00130-8

Cobham, V. E., Filus, A., \& Sanders, M. R. (2017). Working with parents to treat anxiety-disordered children: a proof of concept RCT evaluating Fear-less Triple P. Behaviour Research and Therapy, 95, 128-138. https://doi.org/10.1016/j.brat. 2017.06.004

Cohen, J. (1988). Statistical Power Analysis for the Behavioural Sciences (2nd edn). Lawrence Earlbaum Associates.

Collins, P. Y., Patel, V., Joestl, S. S., March, D., Insel, T. R., Daar, A. S., Bordin, I.A., Costello, E. J., Durkin, M., Fairburn, C., Glass, R. I., Hall., W., Huang, Y., Hyman, S. E., Jamison, K., Syvlia, K., Kapur, S., Kleinman, A., Ogunniyi, A., ... \& Wolport, M. (2011). Grand challenges in global mental health. Nature, 475, 27-30. https://doi.org/10.1038/475027a

Costello, E. J., Erklani A., \& Angold, A. (2006). Is there an epidemic of child or adolescent depression? Journal of Child Psychology \& Psychiatry, 47, 1263-1271. https://doi.org/10.1111/j.1469-7610.2006.01682.x

Department of Health \& Department of Education (2017). Transforming children and young people's mental health provision: a green paper. https://assets.publishing.service.gov.uk/government/uploads/system/uploads/attachment_data/ file/664855/Transforming_children_and_young_people_s_mental_health_provision.pdf

Department of Health \& Department of Education (2018). Government response to the consultation on "Transforming children and young people's mental health provision: a green paper" and next steps. https://assets.publishing.service. gov.uk/government/uploads/system/uploads/attachment_data/file/728892/government-response-to-consultation-ontransforming-children-and-young-peoples-mental-health.pdf

Department of Health CYP Mental Health \& Wellbeing Taskforce (2015). Future in mind: promoting, protecting and improving our children and young people's mental health and wellbeing. NHS England. https://assets.publishing.service. gov.uk/government/uploads/system/uploads/attachment_data/file/414024/Childrens_Mental_Health.pdf

Eckshtain, D., Kuppens, S., Ugueto, A., Ng, M. Y., Vaughn-Coaxum, R., Corteselli, K., \& Weisz, J. R. (2020). Meta-analysis: 13-year follow-up of psychotherapy effects on youth depression. Journal of the American Academy of Child \& Adolescent Psychiatry, 59, 45-63. https://doi.org/10.1016/j.jaac.2019.04.002

Essau, C. A., Lewinsohn, P. M., Olaya, B., \& Seeley, J. R. (2014). Anxiety disorders in adolescents and psychosocial outcomes at age 30. Journal of Affective Disorders, 163, 125-132. https://doi.org/10.1016/j.jad.2013.12.033

Ewing, D. L., Monsen, J. J., Thompson, E. J., Cartwright-Hatton, S., \& Field, A. (2015). A meta-analysis of transdiagnostic cognitive behavioural therapy in the treatment of child and young person anxiety disorders. Behavioural and Cognitive Psychotherapy, 43, 562-577. https://doi.org/10.1017/S1352465813001094

Fancourt, D., Steptoe, A., \& Bu, F. (2021). Trajectories of anxiety and depressive symptoms during enforced isolation due to COVID-19 in England: a longitudinal observational study. The Lancet Psychiatry, 8, 141-149. https://doi.org/10.1016/ S2215-0366(20)30482-X

Fonagy, P., Cottrell, D., Phillips, J., Bevington, D., Glaser, D., \& Allison, E. (2015). What Works for Whom? A Critical Review of Treatments for Children and Adolescents (2nd edn). London, UK: Guilford Press

Ford, T., Goodman, R., \& Meltzer, H. (2003). The British child and adolescent mental health survey 1999: the prevalence of DSM-IV disorders. Journal of the American Academy of Child \& Adolescent Psychiatry, 42, 1203-1211. https://doi.org/10. 1097/00004583-200310000-00011

Ford, T., Hamilton, H., Meltzer, H., \& Goodman, R. (2007). Child mental health is everybody's business: the prevalence of contact with public sector services by type of disorder among British school children in a three-year period. Child \& Adolescent Mental Health, 12, 13-20. https://doi.org/10.1111/j.1475-3588.2006.00414.x

Frith, E. (2017). Access and Waiting Times for Children and Young People's Mental Health Services. Education Policy Institute. https://allcatsrgrey.org.uk/wp/download/public_health/mental_health/EPI_Access-and-waiting-times_.pdf

Fuggle, P., \& Hepburn, C. (2019). Clinical outcomes for the wellbeing practitioner programme for children, young people, and their parents/carers: update report December 2019. Anna Freud National Centre for Children and Families. https://manuals. annafreud.org/cwp/\#National\%20CWP\%20Outcomes\%20Report\%202019

Gallagher, H. M., Rabian, B. A., \& McCloskey, M. S. (2004). A brief group cognitive-behavioral intervention for social phobia in childhood. Journal of Anxiety Disorders, 18, 459-479. https://doi.org/10.1016/S0887-6185(03)00027-6

Ginsburg, G. S., \& Drake, K. L. (2002). School-based treatment for anxious African-American adolescents: a controlled pilot study. Journal of the American Academy of Child \& Adolescent Psychiatry, 41, 768-775. https://doi.org/10.1097/00004583200207000-00007

Goodman, R. (2001). Psychometric properties of the strengths and difficulties questionnaire. Journal of the American Academy of Child and Adolescent Psychiatry, 40, 1337-1345. https://doi.org/10.1097/00004583-200111000-00015

Green, H., McGinnity, A., Meltzer, H., Ford, T., \& Goodman, R. (2004). Mental health of children and young people in Great Britain, 2004. Office for National Statistics. http://doc.ukdataservice.ac.uk/doc/5269/mrdoc/pdf/5269technicalreport.pdf

Greenberger, D., \& Padesky, C. A. (2016). Mind over Mood: Change How You Feel by Changing the Way you Think (2nd edn). New York, USA: Guilford Press

Harden, A., Rees, R., Shepherd, J., Brunton, G., Oliver, S. \& Oakley, A. (2001). Young people and mental health: a systematic review of research on barriers and facilitators. London: EPPI-Centre, Social Science Research Unit, Institute of Education, 
University of London. https://eppi.ioe.ac.uk/cms/Portals/0/PDF\%20reviews\%20and\%20summaries/mental_health.pdf? ver $=2006-03-02-124527-247$

Health Education England (2017). Stepping forward to 2020/2021. The mental health workforce plan for England. NHS England. https://www.hee.nhs.uk/sites/default/files/documents/Stepping\%20forward\%20to\%20202021\%20-\%20The\% 20mental\%20health\%20workforce\%20plan\%20for\%20england.pdf

Holmes, M. C., Donovan, C. L., Farrell, L. J., \& March, S. (2014). The efficacy of a group-based, disorder-specific treatment program for childhood GAD - a randomized controlled trial. Behaviour Research and Therapy, 61, 122-135. https://doi. org/10.1016/j.brat.2014.08.002

House of Commons Health Committee (2014). Children's and adolescents' mental health and CAMHS: third report of session 2014-15. House of Commons. https://publications.parliament.uk/pa/cm201415/cmselect/cmhealth/342/342.pdf

Independent Mental Health Taskforce (2016). Five year forward view for mental health. NHS England. https://www.england. nhs.uk/wp-content/uploads/2016/02/Mental-Health-Taskforce-FYFV-final.pdf

James, I. A., Blackburn, I. M., \& Reichelt, F. K. (2001). Manual of the Revised Cognitive Therapy Scale (CTS-R). https://www. ed.ac.uk/files/atoms/files/ctsrmanual.pdf

James, A. C., Reardon, T., Soler, A., James, G., \& Creswell, C. (2020). Cognitive behavioural therapy for anxiety disorders in children and adolescents. Cochrane Database of Systematic Reviews. https://doi.org/10.1002/14651858.CD013162.pub2

Johnstone, K.M., Kemps, E. \& Chen, J. (2018). A meta-analysis of universal school-based prevention programs for anxiety and depression in children. Clinical Child and Family Psychology Review, 21, 466-481. https://doi.org/10.1007/s10567-0180266-5

Kessler, R. C., Chiu, W. T. Demler, O., Walters, E. E. (2005). Prevalence, severity, and comorbidity of twelve-month DSM-IV disorders in the national comorbidity survey replication (NCS-R). 'Archives of General Psychiatry, 62, 617-627. https://doi.org/10.1001/archpsyc.62.6.617

Kessler, R. C., Angermeyer, M., Anthony, J. C., Graaf, R. D., Demyttenaere, K. Gasquet, I., De Girolamo, G., Gluzman, S., Gureje, O., Haro, J., Kawakami, N., Karam, A., Levinson, D., Mora, M. E. M., Oakley Browne, M. A., Posada-Villa, J., Stein, D. J., Adley Tsang, C. H., Aguilar-Gaxiola, S., . . \& Ustun, T. B. (2007). Lifetime prevalence and age-of-onset distributions of mental disorders in the World Health Organization's World Mental Health Survey Initiative. World Psychiatry, 6, 168-176.

Kim-Cohen, J., Caspi, A., Moffitt, T. E., Harrington, H., Milne, B. J., \& Poulton, R. (2003). Prior juvenile diagnoses in adults with mental disorder: developmental follow-back of a prospective-longitudinal cohort. Archives of General Psychiatry, 60, 709-717. https://doi.org/10.1001/archpsyc.60.7.709

Kitchen, C. E., Tiffin, P. A., Lewis, S., Gega, L., \& Ekers, D. (2020). Innovations in practice: a randomised controlled feasibility trial of behavioural activation as a treatment for young people with depression. Child and Adolescent Mental Health. Advanced online publication. https://doi.org/10.1111/camh.12415

Kuyken, W., Padesky, C. A., \& Dudley, R. (2011). Collaborative Case Conceptualization: Working Effectively with Clients in Cognitive-Behavioral Therapy. London, UK: Guilford Press.

Law, D., \& Wolpert, M. (2014). Guide to Using Outcomes and Feedback Tools with Children, Young People and Families (2nd edn). Child Outcomes Research Consortium (CORC) Ltd. https://www.corc.uk.net/media/2112/201404guide_to_using outcomes_measures_and_feedback_tools-updated.pdf

Levita, L. (2020). Initial research findings on the impact of COVID-19 on the well-being of young people aged 13 to 24 in the UK: Report 1. COVID-19 Psychological Research Consortium (C19PRC). https://www.rcpch.ac.uk/sites/default/files/2020-08/ Impact $\% 20$ of $\% 20$ COVID-19\%20on\%20the\%20well-being\%20of\%20young\%20people\%20aged\%2013\%20to\%2024\%20\%20University\%20of\%20Sheffield.pdf

Liddle, I., \& Macmillan, S. (2010). Evaluating the FRIENDS programme in a Scottish setting. Educational Psychology in Practice, 26, 53-67. https://doi.org/10.1080/02667360903522785

March, J. S., \& Vitiello, B. (2009). Clinical messages from the treatment of adolescents with depression study (TADS). American Journal of Psychiatry, 166, 1118-1123. https://doi.org/10.1176/appi.ajp.2009.08101606

Martell, C. R., Addis, M. E., \& Jacobson, N. S. (2001). Depression in Context: Strategies for Guided Action. Norton.

Martin, F., \& Oliver, T. (2019). Behavioral activation for children and adolescents: a systematic review of progress and promise. European Child \& Adolescent Psychiatry, 28, 427-441. https://doi.org/10.1007/s00787-018-1126-Z

Mavji, D., Rees, J., Venture, E., Parnell, N., Barker, H., Bolton, D., Williams, S., Ellis, L., Fuggle, P., Geraghty, W., Law, D., \& Fuggle, T. (2018). London and South East CYP-IAPT collaborative children's wellbeing practitioner (CWP programme): audit report for cohort 1 2017-2018. https://manuals.annafreud.org/cwp/\#Audit\%20of\%20the\%20CWP\% 20Programme\%20in\%20London\%20and\%20the\%20South\%20East

McCauley, E., Schloredt, K., Gudmundsen, G., Martell, C., \& Dimidjan, S. (2011). Expanding behavioural activation to depressed adolescents: lessons learned in treatment development. Cognitive \& Behavioural Practice, 18, 371-383. https://doi.org/10.1016/j.cbpra.2010.07.006

McCauley, E., Gudmundsen, G., Schloredt, K., Martell, C., Rhew, I., Hubley, S., \& Dimidjian, S. (2016). The adolescent behavioral activation program: adapting behavioral activation as a treatment for depression in adolescence. Journal of Clinical Child and Adolescent Psychology, 45, 291-304. https://doi.org/10.1080/15374416.2014.979933 
McConachie, H., McLaughlin, E., Grahame, V., Taylor, H., Honey, E., Tavernor, L., Rodgers, J., Freeston, M., Hemm, C., Steen, N., \& Le Couteur, A. (2014). Group therapy for anxiety in children with autism spectrum disorder. Autism, 18, 723-732. https://doi.org/10.1177\%2F1362361313488839

McLoone, J. K., \& Rapee, R. M. (2012). Comparison of an anxiety management program for children implemented at home and school: lessons learned. School Mental Health, 4, 231-242. https://doi.org/10.1007/s12310-012-9088-7

Melfsen, S., Kühnemund, M., Schwieger, J., Warnke, A., Stadler, C., Poustka, F., \& Stangier, U. (2011). Cognitive behavioral therapy of socially phobic children focusing on cognition: a randomised wait-list control study. Child and Adolescent Psychiatry and Mental Health, 5. https://doi.org/10.1186/1753-2000-5-5

Mifsud, C., \& Rapee, R. M. (2005). Early intervention for childhood anxiety in a school setting: outcomes for an economically disadvantaged population. Journal of the American Academy of Child and Adolescent Psychiatry, 44, 996-1004. https://doi. org/10.1097/01.chi.0000173294.13441.87

Munoz-Solomondo, A., Kendall, T., \& Whittington, C. (2008). Cognitive behavioural therapy for children and adolescents. Current Opinion in Psychiatry, 21, 332-337. https://doi.org/10.1097/YCO.0b013e328305097c

Murphy, M., \& Fonagy, P. (2012). Mental health problems in children and young people. In Chief Medical Officer Annual Report, Our Children Deserve Better: Prevention Pays (ch. 10). Department of Health \& Social Care.

National Institute for Health \& Care Excellence (2019). Depression in children and young people: identification and management. NICE guideline [NG134]. https://www.nice.org.uk/guidance/ng134

NHS (2019). The NHS long term plan. https://www.longtermplan.nhs.uk/

NHS Digital (2020). Waiting times for children and young people's mental health services, 2019-20. [Data set]. https://digital. nhs.uk/data-and-information/supplementary-information/2020/waiting-times-for-children-and-young-peoples-mentalhealth-services-2019-2020-additional-statistics

Olivares-Olivares, P. J., Ortiz-González, P. F., \& Olivares, J. (2019). Role of social skills training in adolescents with social anxiety disorder. International Journal of Clinical and Health Psychology, 19, 41-48. https://doi.org/10.1016/j. ijchp.2018.11.002

Oud, M., De Winter, L., Vermeulen-Smit, E., Bodden, D., Nauta, M., Stone, L., van den Heuvel, M., Al Taher, R., de Graaf, I., Kendall, T., Engels, R., \& Stikkelbroek, Y. (2019). Effectiveness of CBT for children and adolescents with depression: a systematic review and meta-regression analysis. European Psychiatry, 57, 33-45. https://doi.org/10. 1016/j.eurpsy.2018.12.008

Pass, L., Brisco, G., \& Reynolds, S. (2015). Adapting brief behavioural activation (BA) for adolescent depression: a case example. the Cognitive Behaviour Therapist, 8, E17. https://doi.org/10.1017/S1754470X15000446

Pass, L., Hodgson, E., Whitney, H., \& Reynolds, S. (2018a). Brief behavioral activation treatment for depressed adolescents delivered by nonspecialist clinicians: a case illustration. Cognitive and Behavioral Practice, 25, 208-224. https://doi.org/10. 1016/j.cbpra.2017.05.003 3

Pass, L., Lejuez, C. W., \& Reynolds, S. (2018b). Brief behavioural activation (brief BA) for adolescent depression: a pilot study. Behavioural and Cognitive Psychotherapy, 46, 182-194. https://doi.org/10.1017/S1352465817000443

Pass, L., Sancho, M., Brett, S., Jones, M., \& Reynolds, S. (2018c). Brief behavioural activation (Brief BA) in secondary schools: a feasibility study examining acceptability and practical considerations. Educational and Child Psychology, 35, 10-20. http://centaur.reading.ac.uk/77759/

Pass, L., Whitney, H., \& Reynolds, S. (2016). Brief behavioral activation for adolescent depression: working with complexity and risk. Clinical Case Studies, 15, 360-375. https://doi.org/10.1177/1534650116645402

Podell, J. L., Kendall, P. C., Gosch, E. A., Compton, S. N., March, J. S., Albano, A. M., Rynn, M. A., Walkup, J. T., Sherrill, J. T., Ginsburg, G. S., Keeton, C. P., Birmaher, B. \& Piacentini, J. C. (2013). Therapist factors and outcomes in CBT for anxiety in youth. Professional Psychology: Research and Practice, 44, 89-98. https://doi.org/10. 10337/a0031700

Rapee, R. M., Abbott, M. J., \& Lyneham, H. J. (2006a). Bibliotherapy for children with anxiety disorders using written materials for parents: a randomized controlled trial. Journal of Consulting and Clinical Psychology, 74, 436-444. https://psycnet.apa.org/doi/10.1037/0022-006X.74.3.436

Rapee, R. M., Lyneham, H. J., \& Schniering, C. A. (2006b). Cool Kids (Chilled) Adolescent Anxiety and Depression Program. Sydney, Australia: Centre for Emotional Health, Macquarie University.

Rasing, S., Creemers, D. H., Janssens, J. M., \& Scholte, R. H. (2017). Depression and anxiety prevention based on cognitive behavioral therapy for at-risk adolescents: a meta-analytic review. Frontiers in Psychology, 8, 1066. https://doi.org/10.3389/ fpsyg.2017.01066

Raw, J., Waite, P., Pearcey, S., Creswell, C., Shum, A., \& Patalay, P. (2021). Examining changes in parent-reported child and adolescent mental health throughout the UK's first COVID-19 national lockdown. PsyArXiv. https://doi.org/10.31234/osf.io/ exktj

Reardon, T., Harvey, K., \& Creswell, C. (2019). Seeking and accessing professional support for child anxiety in a community sample. European Child \& Adolescent Psychiatry, 29, 649-664. https://doi.org/10.1007/s00787-019-01388-4

Rees, G., Bradshaw, J., Goswami, H., \& Keung, A. (2008). Understanding children's wellbeing: a national survey of young people's wellbeing. The Children's Society. https://www.york.ac.uk/inst/spru/research/pdf/Understanding.pdf 
Richards, D. A., \& McDonald, B. (1990). Behavioural Psychotherapy: A Handbook for Nurses. Oxford, UK: Heinemann Nursing

Roth, A., \& Fonagy, P. (2005). What Works for Whom? A Critical Review of Psychotherapy Research. London, UK: Guilford Press.

Sadler, K., Vizard, T., Ford, T., Goodman, A., Goodman, R., \& McManus, S. (2018). Mental health of children and young people in England, 2017. NHS Digital. https://digital.nhs.uk/data-and-information/publications/statistical/mental-healthof-children-and-young-people-in-england/2017/2017

Safran, J. D., \& Segal, Z. V. (1990). Interpersonal Process in Cognitive Therapy. New York, USA: Basic Books.

Simon, E., Bögels, S. M., \& Voncken, J. M. (2011). Efficacy of child-focused and parent-focused interventions in a child anxiety prevention study. Journal of Clinical Child \& Adolescent Psychology, 40, 204-219. https://doi.org/10.1080/ 15374416.2011.546039

Stallard, P. (2002). Think Good-Feel Good. A Cognitive Behavioural Workbook for Children and Young People. Chichester, UK: John Wiley \& Sons Limited

Scott, S. (2018). A national approach to improving child and adolescent mental health care: The children and young people's improving access to psychological therapies program in England. In J. R. Weisz \& A. E. Kazdin (Eds.), Evidence-based psychotherapies for children and adolescents (3rd ed. pp. 415-429). London: The Guildford Press.

Shum, A., Skripkauskaite, S., Pearcey, S., Waite, P., \& Creswell, C. (2021). Report 09: Update on children's \& parents/carers' mental health; Changes in parents/carers' ability to balance childcare and work: March 2020 to February 2021. Co-SPACE study, University of Oxford, University College London, and University of Leicester. http://cospaceoxford.org/wp-content/ uploads/2021/03/Report_09_15march2021.pdf

Stallard, P., Skryabina, E., Taylor, G., Phillips, R., Daniels, H., Anderson, R., \& Simpson, N. (2014). Classroom-based cognitive behaviour therapy (FRIENDS): a cluster randomised controlled trial to Prevent Anxiety in Children through Education in Schools (PACES). The Lancet Psychiatry, 1, 185-192. https://doi.org/10.1016/s2215-0366(14)70244-5

Stockings, E., Degenhardt, L., Dobbins, T., Lee, Y., Erskine, H., Whiteford, H., \& Patton, G. (2016). Preventing depression and anxiety in young people: a review of the joint efficacy of universal, selective and indicated prevention. Psychological Medicine, 46, 11-26. http://doi.org/10.1017/S0033291715001725

Thirlwall, K., Cooper, P. J., Karalus, J., Voysey, M., Willetts, L., \& Creswell, C. (2013). Treatment of child anxiety disorders via guided parent-delivered cognitive-behavioural therapy: randomised controlled trial. British Journal of Psychiatry, 203, 436-444. https://doi.org/10.1192/bjp.bp.113.126698

Tindall, L., Mikocka-Walus, A., McMillan, D., Wright, B., Hewitt, C., \& Gascoyne, S. (2017). Is behavioural activation effective in the treatment of depression in young people? a systematic review and meta-analysis. Psychology and Psychotherapy: Theory, Research and Practice, 90, 770-796. https://doi.org/10.1111/papt.12121

Vizard, T., Sadler, K., Ford, T., Newlove-Delgado, T., McManus, S., Marcheselli, F., Davis, J., Williams, T., Leach, C., Mandalia, D., \& Cartwright, C. (2020). Mental health of children and young people in England, 2020 Wave 1 follow up to the 2017 survey. NHS Digital. https://igital.nhs.uk/data-and-information/publications/statistical/mental-health-ofchildren-and-young-people-in-england/2020-wave-1-follow-up

Waite, P. D., Pearcey, S., Shum, A., Raw, J., Patalay, P., \& Creswell, C. (2020). How did the mental health of children and adolescents change during early lockdown during the COVID-19 pandemic in the UK? PsyArXiv. https://doi.org/10.31234/ osf.io/t8rfx

Wang, Z., Whiteside, S., Sim, L., Farah, W., Morrow, A. S., Alsawas, M., Barrionuevo, P., Tello, M., Asi, N., Beuschel, B., Daraz, L., Almasri, J., Zaiem, F., Larrea-Mantilla, L., Ponce, O. J., LeBlanc, A., Prokop, L. J., \& Murad, M. H. (2017). Comparative effectiveness and safety of cognitive behavioral therapy and pharmacotherapy for childhood anxiety disorders: a systematic review and meta-analysis. JAMA Pediatrics, 171, 1049-1056. https://doi.org/10.1001/ jamapediatrics.2017.3036

Warwick, H., Reardon, T., Cooper, P., Murayama, K., Reynolds, S., Wilson, C., \& Cresswell, C. (2017). Complete recovery from anxiety disorders following cognitive behavior therapy in children and adolescents: a meta-analysis. Clinical Psychology Review, 52, 77-91. https://doi.org/10.1016/j.cpr.2016.12.002

Weisz, J. R., McCarty, C. A., \& Valeri, S. M. (2006). Effects of psychotherapy for depression in children and adolescents: a meta-analysis. Psychological Bulletin, 132, 132-149. https://doi.org/10.1037/0033-2909.132.1.132

Werner-Seidler, A., Perry, Y., Calear, A. L., Newby, J. M., \& Christensen, H. (2017). School-based depression and anxiety prevention programs for young people: a systematic review and meta-analysis. Clinical Psychology Review, 51, 30-47. https://doi.org/10.1016/j.cpr.2016.10.005

Cite this article: Lockhart G, Jones C, and Sopp V. A pilot practice-based outcomes evaluation of low-intensity cognitive behavioural interventions delivered by postgraduate trainees to children and young people with mild to moderate anxiety or low mood: an efficient way forward in mental health care? The Cognitive Behaviour Therapist. https://doi.org/10.1017/ S1754470X21000301 